\title{
Utility-Proportional Fairness in Wireless Networks
}

\author{
G. Tychogiorgos, A. Gkelias and K. K. Leung \\ Electrical and Electronic Engineering Department \\ Imperial College, London SW7 2AZ, UK \\ \{g.tychogiorgos, a.gkelias, kin.leung\}@imperial.ac.uk
}

\begin{abstract}
Current communication networks support a variety of applications with different quality of service (QoS) requirements which compete for its resources. This continuously increasing competition highlights the necessity for more efficient and fair resource allocation. Current Network Utility Maximization (NUM) framework fails to achieve this target and alternative approaches cannot operate in networks that consist of wireless links. This paper presents a NUM framework for wireless networks that shares resources according to the utility proportional fairness policy. This policy is shown to prevent rate oscillations in the resource allocation process, allocate resources in a more fair manner among different types of applications and lead to the calculation of closed form solutions for the optimal rate allocation function. Based on this policy, a distributed rate and power allocation algorithm is proposed that gives priority to applications with greater need of resources. Finally, numerical results on the performance of the proposed algorithm are presented and compared against other approaches in the literature.
\end{abstract}

\section{INTRODUCTION}

Since the seminal papers of Kelly et al. [1] and Low et al. [2], the proposed Network Utility Maximization (NUM) framework has found numerous applications in communication networks since it made clear that expressing the network resource allocation as an optimization problem can be solved by low-complexity distributed algorithms. More specifically, they proposed an optimization problem of the form:

$$
\begin{aligned}
& \text { Problem } \Pi_{\mathrm{NUM}}: \max \\
& \sum_{r \in R} U_{r}\left(x_{r}\right) \\
& \text { s. t. } \quad \boldsymbol{A} x \leq \boldsymbol{C}, \quad x \geq 0,
\end{aligned}
$$

where $r$ denotes the index of the source node, $x_{r}$ represents the transmission rate of node $r, C$ is a vector containing the capacities of all links and $U_{r}\left(x_{r}\right)$ is the utility of node $r$ when transmitting at rate $x_{r}$. In essence, the utility represents the degree of satisfaction of a user as a function of the transmission rate. Moreover, $A_{j r}$, the element of matrix $\boldsymbol{A}$, is 1 when link $j$ lies on route $r$, and 0 otherwise. The authors propose an iterative distributed algorithm where each link in the network charges for its resources and the users determine their transmission rates according to the maximum amount they are willing to pay. This algorithm optimizes the resource

Research was partially sponsored by the U.S. Army Research Laboratory and the U.K. Ministry of Defence and was accomplished under Agreement Number W911NF-06-3-0001. The views and conclusions contained in this document are those of the author(s) and should not be interpreted as representing the official policies of the U.S. Army Research Laboratory, the U.S Government, the U.K. Ministry of Defence or the U.K. Government. The U.S and U.K. Governments are authorized to reproduce and distribute reprints for Government purposes notwithstanding any copyright notation hereon. allocation under two major assumptions; the utilities are all concave functions of rate and all links have fixed capacity, e.g. are wired. These two assumptions are responsible for a number of shortcomings of current NUM approaches, which will be discussed in detail in the remainder of this section.

Concave utilities are ideal to model applications that generate elastic traffic [3]. Elasticity describes an application's ability to adapt easily to changes in the network conditions, such as delay, throughput etc, while still meeting some QoS requirements. Examples of such applications, include FTP and HTTP [4][5][6], which used to generate the majority of the traffic in the internet until recently. However, the majority of the traffic in current networks is generated by realtime applications ${ }^{1}$ that are considered inelastic. Existing work models such applications using non-concave sigmoidal utility functions [8][9][10] that turn the resulting formulation into a non-convex problem. An example of such utility function is shown at the top subplot in Figure 1. Despite the existence of analytic methodology to solve or approximate the optimal solution for such problems in a distributed way, this approach has significant disadvantages:

- The optimal rate allocation function of a source node, $x_{r}^{*}\left(\lambda^{r}\right)^{2}$, is hard to be calculated in a closed form and therefore numerical gradient-based approaches must be used, which however increase convergence time and degrade accuracy.

- Function $x_{r}^{*}\left(\lambda^{r}\right)$ is discontinuous for some values of link price. This causes oscillations in the network that can prevent the algorithm from converging. An example of $x_{r}^{*}\left(\lambda^{r}\right)$ for a sigmoidal utility function is shown in blue at the bottom subplot of Figure 1.

- The heuristics proposed in literature to resolve these oscillations offer approximations that in some cases can be far from the optimal solution.

- Despite the fact that the utility function $U_{r}\left(x_{r}\right)$ is defined for rates within the range $\left[x_{r}^{\min }, x_{r}^{\max }\right]$, only a small part of this range can be achieved. This restricts the applicability of such approaches in practical problems. For example, the rate for the utility of Figure 1 takes values within the range $[0,10]$ but the feasible range region (shown in black) is restricted only to either zero or values within $[6.51,10]$.

The traditional NUM formulation maximizes the aggregate utility in the network. Moreover, it has been shown [1]

\footnotetext{
${ }^{1}$ and their percentage is expected to increase as forecasted in [7]

${ }^{2}$ where $\lambda^{r}$ is the aggregate price in the network
} 

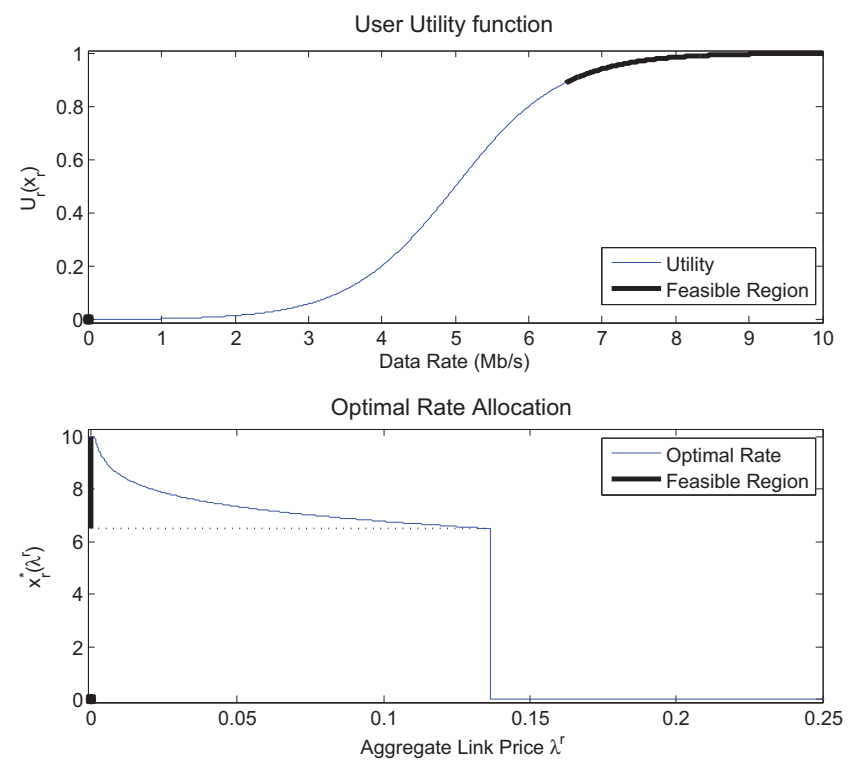

Fig. 1. The feasible rate region of a sigmoidal utility function

that the resulting bandwidth allocations follows the so-called (bandwidth) proportional fairness. While this type of fairness seems to perform well when all users follow the same utility, this approach is responsible for some contradictory behaviors in cases that users follow different utilities, i.e. when users have different QoS needs. In such cases, proportional fairness favors users which require low rate to achieve high utility. As pointed out first in [11], a bandwidth proportional fair optimization algorithm favors users with low demand, i.e. those with rapidly increasing utility function. This happens because allocating a unit of rate to a utility with large derivative leads to larger increase in the aggregate utility than when allocating to users with high demand, i.e. with small value of utility derivative. To resolve this contradictory behavior, authors in [11] define a new type of fairness, called utility proportional fairness. According to that, a bandwidth allocation $\boldsymbol{x}^{*}=\left[x_{1}, x_{2}, \ldots, x_{R}\right]^{T}$ is utility proportional fair, if it is feasible and for any other feasible allocation $\boldsymbol{x}$,

$$
\sum_{r \in R} \frac{x_{r}-x_{r}^{*}}{U_{r}\left(x_{r}^{*}\right)} \leq 0 .
$$

The utility proportional fairness can be achieved if the utility function of each user is transformed according to:

$$
\mathcal{U}_{r}\left(x_{r}\right)=\int_{m_{r}}^{x_{r}} \frac{1}{U_{r}(y) d y}, \quad m_{r} \leq x_{r} \leq M_{r},
$$

where $m_{r}$ and $M_{r}$ are the minimum and maximum transmission rates for user $r$ respectively, and the objective function of Problem $\Pi_{\text {NUM }}$ is changed to $\sum_{r \in R} \mathcal{U}_{r}\left(x_{r}\right)$.

Authors in [11] propose a distributed algorithm to solve Problem $\Pi_{\mathrm{NUM}}$ in order to achieve utility proportional fairness in wired networks shared by various types of applications. However, current communication networks are often consisted of wireless networks, whose capacity is not constant but depends on the interference. This need highlights the necessity of extending the current utility proportional fairness framework to be able to adjust link powers according to the channel conditions in the network.
Motivated by the aforementioned shortcomings of current bandwidth and utility proportional fairness mechanisms in wireless networks, this paper makes the following contributions:

- Proposes a utility proportional fair optimization formulation for high-SINR wireless networks. Utility proportional fairness can prevent the oscillations, caused when a utility function is non-concave, allow the use of the full range of possible rate values and calculate the optimal rate.

- Derives analytical solutions for the optimal rate allocation function for a number of widely used application types.

- Proposes a distributed utility proportional fair algorithm to jointly optimize transmission powers and data rates in high-SINR wireless networks.

The rest of the paper is organized as follows. First, Section II presents a utility proportional fair formulation for highSINR wireless networks and proposes a distributed gradient algorithm to calculate the optimal resource allocation. Consequently, Section III provides closed form solutions of the optimal rate for a number of application types and discusses how these formulas can be used to prevent oscillations. Section IV presents numerical results illustrating the convergence and performance of the proposed approach and Section $\mathrm{V}$ concludes our current work and outlines our future research plan.

\section{PROBLEM Formulation}

This chapter focuses on the development of an optimization formulation for wireless networks that achieves utility proportional fairness while taking into account the interference among wireless links and the different QoS requirements of various applications.

\section{A. Network Model}

Consider a multi-hop wireless network where each node can operate either as traffic source, destination or relay that just forwards traffic to its neighbors. We define the transmission rate vector $\boldsymbol{r}=\left[r_{1}, r_{2}, \ldots, r_{M}\right]^{T}$ which includes the transmission rates of all $M$ source nodes in the wireless network. Moreover, we define the link $l$ as the tuple $\left(T_{l}, R_{l}\right)$, where $T_{l}$ is the transmitting and $R_{l}$ the receiving node, respectively. We also define $\boldsymbol{p}=\left[p_{1}, p_{2}, \ldots, p_{L}\right]^{T}$ as the vector which includes the transmission powers of the $L$ links. The wireless channel is modelled as follows. Let $G$ be a matrix of size $L \times L$, where $G_{k m}$, with $k, m \in 1,2, \ldots, L$, represents the path loss coefficient for the path between the transmitter of link $k$ and the receiver of link $m$. The elements of the path loss matrix $G$ depend on the physical characteristics of the wireless links.

As explained earlier, each source node $i$ is associated with a utility function $U_{i}\left(r_{i}\right)$. The utility function of a user represents the degree of satisfaction that a user enjoys when sending at a specific rate. In other words, the user utility function reflects the Quality of Experience (QoE) of a user when data content is delivered at a specific rate. This QoE cannot be determined precisely for each user but prior work in the literature [6][8] has identified approximate forms/shapes for 
various applications, such as HTTP, FTP and video streaming applications. Finally, we also associate each wireless link $l$ with a convex cost function $V_{l}\left(p_{l}\right)$. This function represents the cost of using the limited power resources of the wireless channel. The incorporation of this cost function leads towards more energy efficient resource allocations.

\section{B. Optimization Problem}

The network performance optimization is formulated as the following maximization problem:

$$
\begin{aligned}
\text { Problem } \Pi_{\mathbf{M W N}}: \max _{\boldsymbol{r}, \boldsymbol{p}} & \sum_{i=1}^{M} \mathcal{U}_{i}\left(r_{i}\right)-\gamma \sum_{l=1}^{L} V_{l}\left(p_{l}\right) \\
\text { s. t. } & \sum_{i=1}^{M} \alpha_{i l} r_{i} \leq C_{l}(\boldsymbol{p}), \forall \text { links } l
\end{aligned}
$$

where $\mathcal{U}_{i}\left(r_{i}\right)$ is the transformed utility function given by (2) for rate $r_{i}$, parameter $\alpha_{i l}$ is one if the traffic of user $i$ is passing through link $l$, and zero otherwise. The rates $r_{i}$, with $i \in 1,2, \ldots, M$, and powers $p_{l}$, with $l \in 1,2, \ldots, L$, are positive quantities and $\gamma$ is a positive weighting parameter. The capacity of a link follows Shannon's capacity formula, $C_{l}(\boldsymbol{p})=B \cdot \log _{2}\left(1+S I N R_{l}\right)$ and is a function of the Signal to Noise plus Interference Ratio (SINR) at the receiver of the link. This formula is a non-concave function of powers and this might prevent any gradient based algorithm from converging to the optimal power vector. However, under the assumption that $S I N R_{l} \gg 1$, the formula $C_{l}(\boldsymbol{p})=B \log _{2}\left(S I N R_{l}\right)$ can provide a sufficiently accurate approximation of link capacity. Moreover, this function is a concave function of powers [12]. For the remainder of this paper, we assume high SINR environments and therefore the link capacity $C_{l}(\boldsymbol{p})$ will be calculated using this approximation.

Duality Theory [13] provides an efficient methodology to solve optimization problems distributedly. For this reason, one should initially form the Lagrangian function as

$$
\begin{aligned}
& L(\boldsymbol{r}, \boldsymbol{p}, \boldsymbol{\lambda})=\sum_{i=1}^{M}\left\{\mathcal{U}_{i}\left(r_{i}\right)-r_{i} \cdot \lambda^{i}\right\} \\
& +\sum_{l=1}^{L} \lambda_{l} B \log \left(\frac{p_{l} G_{l l}}{\sum_{k \neq l} p_{k} G_{k l}+n_{l}}\right)-\gamma \sum_{l=1}^{L} V_{l}\left(p_{l}\right),
\end{aligned}
$$

where $\lambda^{i}=\sum_{l=1}^{L} \alpha_{i l} \lambda_{l}$ is the aggregate price of user $i$ to send a unit of rate through the network.

It is evident from (3) that Problem $\Pi_{\mathrm{MWN}}$ consists of two subproblems coupled by the dual variable vector $\lambda$. The first one determines the optimal rate to maximize the net revenue of the source node, while the second determines the transmission power of the links. Consequently, according to duality theory every source $i$ can calculate its optimal rate $r_{i}^{*}(\boldsymbol{\lambda})$ using

$$
r_{i}^{*}(\boldsymbol{\lambda})=\arg \max \left[\mathcal{U}_{i}\left(r_{i}\right)-r_{i} \cdot \lambda^{i}\right] .
$$

The power and dual variables can be calculated iteratively using:

$$
\lambda_{l}(t)=\lambda_{l}(t-1)-\delta_{\lambda}(t) \frac{\partial L(\boldsymbol{r}, \boldsymbol{p}, \boldsymbol{\lambda})}{\partial \lambda_{l}}
$$

$$
p_{l}(t)=p_{l}(t-1)+\delta_{p}(t) \frac{\partial L(\boldsymbol{r}, \boldsymbol{p}, \boldsymbol{\lambda})}{\partial p_{l}},
$$

where $\delta_{\lambda}(t)$ and $\delta_{p}(t)$ are small positive step sizes and

$$
\begin{gathered}
\frac{\partial L(\boldsymbol{r}, \boldsymbol{p}, \boldsymbol{\lambda})}{\partial \lambda_{l}}=B \cdot \log _{2}\left(\frac{p_{l} G_{l l}}{\sum_{k \neq l} p_{k} G_{k l}+n_{l}}\right)-\sum_{i=1}^{M} \alpha_{i l} r_{i} \\
\frac{\partial L(\boldsymbol{r}, \boldsymbol{p}, \boldsymbol{\lambda})}{\partial p_{l}}=-\gamma V_{l}^{\prime}\left(p_{l}\right)+\frac{1}{p_{l} \ln (2)}\left[\lambda_{l}-\right. \\
\left.\sum_{m \neq l} \lambda_{m} \frac{G_{l m} P_{l}}{\sum_{k \neq m} G_{k m} P_{k}+n_{m}}\right]
\end{gathered}
$$

Equations (4)-(6) constitute a joint primal-dual distributed algorithm, which will be described in detail in the next section along with how utility proportional fairness can lead to the calculation of closed form solutions for (4).

\section{The Price-BAsed Rate Allocation Function}

The existence of various types of user applications complicates the process of calculating the optimal rate allocation of a user for a specific aggregate price. According to optimization theory [14], the optimal rate will be at the point where the first derivative of the objective function diminishes and therefore

$$
r_{i}^{*}(\boldsymbol{\lambda})=\mathcal{U}_{i}^{\prime-1}\left(\lambda^{i}\right) .
$$

In the traditional NUM framework $\mathcal{U}_{i}(\cdot)=U_{i}(\cdot)$, where $U_{i}(\cdot)$ is the utility function of user $i$ as defined earlier. The optimal rate can be calculated using (9) only if the utility function is concave function of rates. If $U_{i}(\cdot)$ is partially convex and partially concave, as with sigmoidal utilities, the first derivative cannot be inverted since it is not a one-to-one function. For sigmoidal utilities, one should use alternative methods with a negative impact on the algorithm convergence speed. Such an alternative could be a gradient based iterative equation of the form:

$$
r_{i}(t+1)=r_{i}(t)+\delta_{r}(t) \frac{\partial L(\boldsymbol{r}, \boldsymbol{p}, \boldsymbol{\lambda})}{\partial r_{i}}
$$

where $\delta_{r}(t)$ is a positive step size and $\frac{\partial L(\boldsymbol{r}, \boldsymbol{p}, \boldsymbol{\lambda})}{\partial r_{i}}$ is the gradient of the lagrangian function with respect to $r_{i}$. However, such an approach will not always converge to the global optimum. In fact, according to the necessary and sufficient condition in [15], the algorithm will converge only if (4) is continuous around the optimal price vector $\boldsymbol{\lambda}$. If this condition does not hold, there can be oscillations in the network that will prevent the algorithm from converging. In such case, an oscillation resolving heuristic, such as the ones presented in [8] and [10], is necessary to ensure stability but at the cost of loosing optimality.

Considering utility proportional fairness, however, by using the transformation of (2), the problem becomes convex even for sigmoidal utilities and (4) always satisfies the condition in [10]. This means that the iterative equation (10) will be able to calculate the optimal solution but more importantly this also allows to calculate a closed form solution for (9) directly. 
TABLE I

The Optimal Resource Allocation Function for Widely Used Types of Applications

\begin{tabular}{|c|l|c|}
\hline Application Type & User Utility Function & Optimal Rate Allocation Function \\
\hline HTTP & $\left.U_{i}\left(r_{i}\right)=\frac{\log \left(\frac{r_{i}}{r^{m i n}}\right)}{\log \left(\frac{r^{m a x}}{r^{m a n}}\right)}\right)$ & $r_{i}^{*}(\boldsymbol{\lambda})=r^{\text {min }} \cdot\left(\frac{r^{\text {max }}}{r^{\text {min }}}\right)^{\frac{1}{\lambda^{i}}}$ \\
\hline FTP & $U_{i}\left(r_{i}\right)=\frac{\log \left(r_{i}+1\right)}{\log \left(r^{\text {max }}+1\right)}$ & $r_{i}^{*}(\boldsymbol{\lambda})=\left(r^{\text {max }}+1\right)^{\frac{1}{\lambda^{i}}}-1$ \\
\hline Video Streaming & $U_{i}\left(r_{i}\right)=\frac{1}{1+\exp \left(-\alpha\left(r_{i}-\beta\right)\right)}$ & $r_{i}^{*}(\boldsymbol{\lambda})=\frac{\alpha \cdot \beta-\log \left(\lambda^{i}-1\right)}{\alpha}$ \\
\hline
\end{tabular}

When the user utility function is transformed according to (2), the first derivative can be easily calculated as:

$$
\mathcal{U}_{i}^{\prime}\left(r_{i}\right)=\frac{1}{U_{i}\left(r_{i}\right)}
$$

Eq. (11) is invertible as long as it is continuous and monotonic, which are both true for any concave utility and any sigmoidal utility that follows the shape shown in Figure 1. Hence, combining (9) and (11), we find that the optimal rate is given by:

$$
r_{i}^{*}(\boldsymbol{\lambda})=U_{i}^{-1}\left(\frac{1}{\lambda^{i}}\right) .
$$

Therefore, it is possible to calculate a closed form solution for (4) for any utility function that satisfies these two properties. This is a significant advantage of the utility proportional fairness approach which leads to the development of algorithms that calculate the optimal solution even for non-concave utilities and converge significantly faster than the traditional approaches.

Based on the analysis above, we derive the optimal rate allocation for browsing, file transfer and video streaming applications using the suggested utility functions in [6] and [8], when utility proportional fairness is applied. These optimal rate allocation functions are demonstrated in Table I. $r^{\text {min }}$ and $r^{\max }$ represent the minimum and maximum transmission rate of a user, and parameters $\alpha$ and $\beta$ are calibration parameters of the sigmoidal utility.

An important observation here is that the continuity of (4) for all aggregate prices also implies that when using utility proportional fairness all rates within the range $\left[r^{\min }, r^{\max }\right]$ are feasible contrary to what happens with bandwidth proportional fairness where only a small part of the total rate range is feasible, as illustrated in Figure 1. This shows that the optimization algorithm has the robustness to adjust to any changes in the link prices and take advantage of the full range of the available rate region in order to maximize user satisfaction in the network.

Having formulated the proportional fair optimization problem for wireless networks and derived closed forms of the optimal rate allocation functions for some of the most common applications, the next step is to develop a distributed algorithm to jointly optimize transmission powers and data rates in the aforementioned wireless network.

The iterative equations (4)-(6) can be used to create a distributed algorithm to jointly optimize rates, powers and prices with minimum information exchange between users. This algorithm consists of two parts; Algorithm 1 is carried

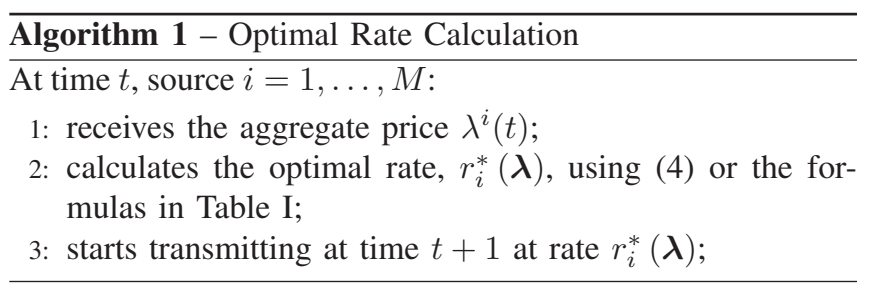

out by each source node and Algorithm 2 in each link. This joint algorithm is an extension of the standard gradientbased algorithm and will converge to the optimal solution for sufficiently small values of the step sizes $\delta_{\lambda}(t)$ and $\delta_{p}(t)$ [14], since Problem $\Pi_{\mathrm{MWN}}$ has been convexified using the utility transformation of (2) and the High-SINR Shannon capacity approximation formula. Regarding the information exchange of the algorithm, users need to know the aggregate link price $\lambda^{i}$. This can be either stored in the ACK packets sent by the destination to the source node, or, if the link price is viewed as the link delay, it can be implicitly measured by the packet queuing delay in the network. Then, the power calculation process requires that a link knows the channel conditions of neighboring nodes. This information can be easily obtained from the lower layers of the protocol stack with no additional signaling overhead.

The use of the cost function $V_{l}\left(p_{l}\right)$ affects convergence of the power control problem as well. When the weighting factor $\gamma$ is zero, there can be a case where equation (8) is always positive. This would cause the distributed algorithm to increase transmission powers indefinitely and therefore the algorithm will not converge. This was further justified in [10] as follows. Consider an arbitrary wireless network and an iteration $t$ of the optimization process where the power vector is $\boldsymbol{p}=\left[p_{l}, l \in\{1, \cdots L\}\right]$ and the rate vector $\boldsymbol{r}=$ $\left[r_{i}^{t}, i \in\{1, \cdots N\}\right]$. If at the next iteration the powers are increased by a small percentage, let $\epsilon$, the resulting power vector will be $\hat{\boldsymbol{p}}=(1+\epsilon) \cdot \boldsymbol{p}$ and the capacity of each link will now become $C_{l}(\hat{\boldsymbol{p}})=B \cdot \log _{2}\left(\frac{(1+\epsilon) \cdot p_{l} G_{l l}}{(1+\epsilon) \cdot \sum_{k \neq l} p_{k} G_{k l}+n_{l}}\right)>C_{l}(\boldsymbol{p})$. This increase of the link capacity would also result in higher network utility and therefore the distributed algorithm will continue increasing powers indefinitely. However, if $\gamma>0$, the optimization algorithm will reach a point where any further increase in the transmission power would not result in an increase at the aggregate utility and therefore the algorithm will converge to a specific power vector.

In existing work, such cases are often prevented by assuming a maximum transmission power. Such an assumption, would be reasonable in practical systems, but is not applicable 

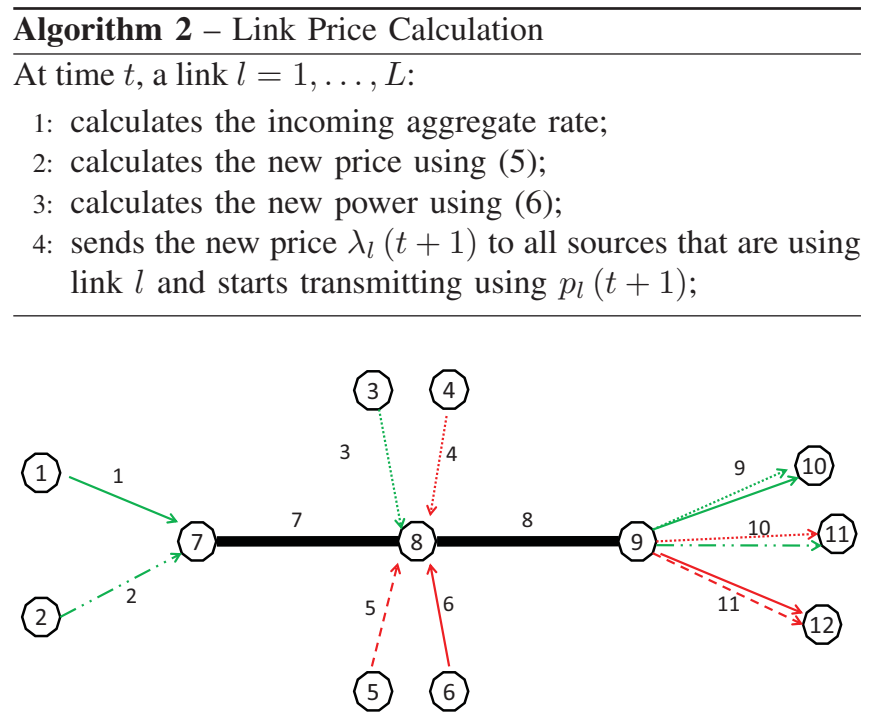

Fig. 2. Network Topology Example

on the theoretical analysis of the problem since it creates artificial convergence points [16]. Therefore, using the cost function $V_{l}\left(p_{l}\right)$ is a more natural way of assuring both energy efficiency and convergence of the distributed power control algorithm

\section{NumericAl RESUlts}

The utility proportional fairness (UPF) approach was applied to various network scenarios in MATLAB, an example of which is the network topology shown in Figure 2 for illustration purposes. The wireless network consists of 6 source nodes, 3 intermediate nodes and a set of 3 destination nodes. The simulation setup consisted of a variety of types of applications, including FTP, HTTP and multimedia applications. This dictated the use of different utility functions, concave or sigmoidal, according to the type of application. All applications were modelled using the utilities of Table I for various values of parameters. More specifically for the example of Figure 2, source nodes 1-3 and 5 serve realtime applications, whereas source nodes 4 and 6 serve elastic applications modelled by concave utilities. The path loss coefficients $G_{l l}$ were significantly larger than these of the interfering channels, i.e. terms $G_{k l}$ for $k=1, \ldots, L$ and $k \neq l$, in order to allow the use of the high-SINR channel capacity approximation formula with low approximation error.

The performance of the UPF approach is compared against the traditional bandwidth proportional fairness (BPF) [8] approach used in prior work in order to show that UPF can successfully avoid the occurence of rate oscillations and can lead to fair allocation of resources when heterogeneous applications compete. During the BFP optimization process, the self-regulating heuristic [8] was used in order to resolve any oscillations that might occur.

Figure 3 shows the convergence of both the objective function of the optimization problem and the utility functions of sources $1-4$. When BPF is used, users 1 and 3 follow a sigmoidal utility and start to oscillate after about 180 iterations, as the spikes indicate. The self-regulating heuristic
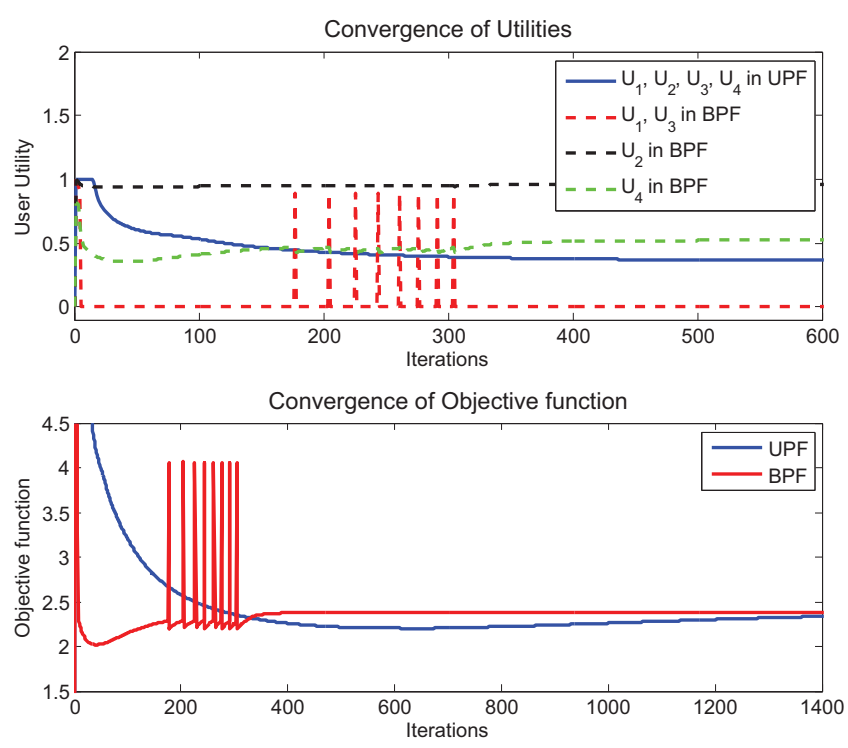

Fig. 3. Convergence of Utility and Objective Functions

removes them from the optimization process and therefore their utility is 0 . The remaining users compete for all the network resources which leads to higher individual utilities for these users. On the other hand, there are no oscillations when UPF is used and the resulting rate allocation leads to the same degree of satisfaction for all sources. In general, UPF gives priority to users with higher rate requirements while BPF allocates more rate to users that are satisfied easier in an attempt to achieve higher aggregate utility in the network. For example, at the final rate allocation in BPF, all the elastic applications are allocated some rate while only two out of the four multimedia applications are allowed to transmit.

The convergence of the rate allocation of the first four sources for both UPF and BPF approaches is illustrated in Figure 4. It is evident that for BPF the oscillations occuring at the rate allocation of sources 1 and 3 cause spikes in the allocations of the rest as well, while in UPF rate are converging
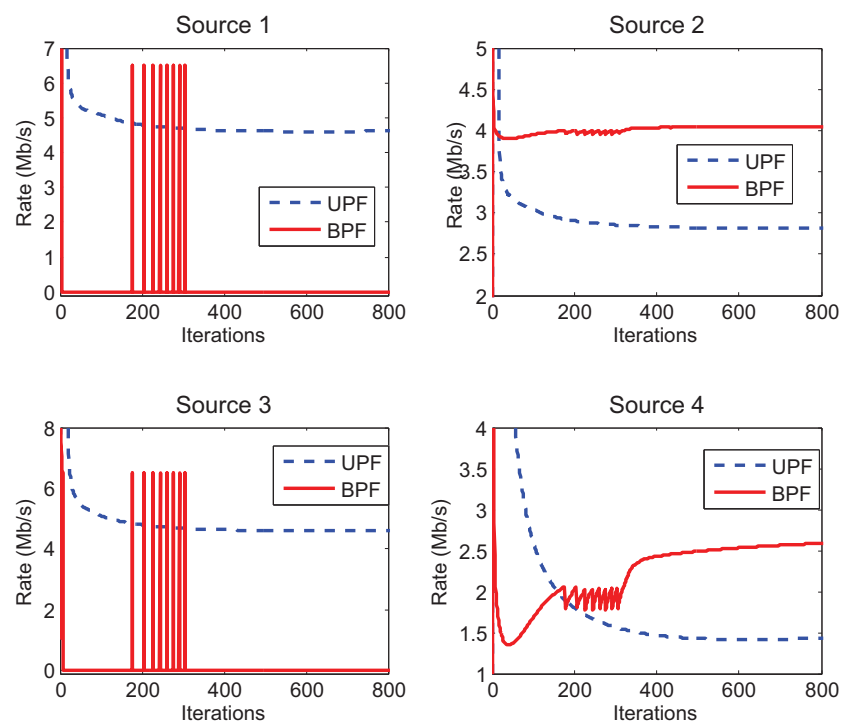

Fig. 4. Convergence of Rate Allocation 
smoothly to the optimal solution. Finally, Figure 5 shows the convergence of the power allocation for links 1 to 4 . It is evident from the peaks around iteration 190 that the existence of oscillations in the BPF approach affects the convergence of powers as well, whereas in UPF the powers converge smoothly to their optimal values. In addition, it is clear that the different allocation policy between UPF and BPF also leads to different values of transmission powers due to the difference in the traffic passing through each link.
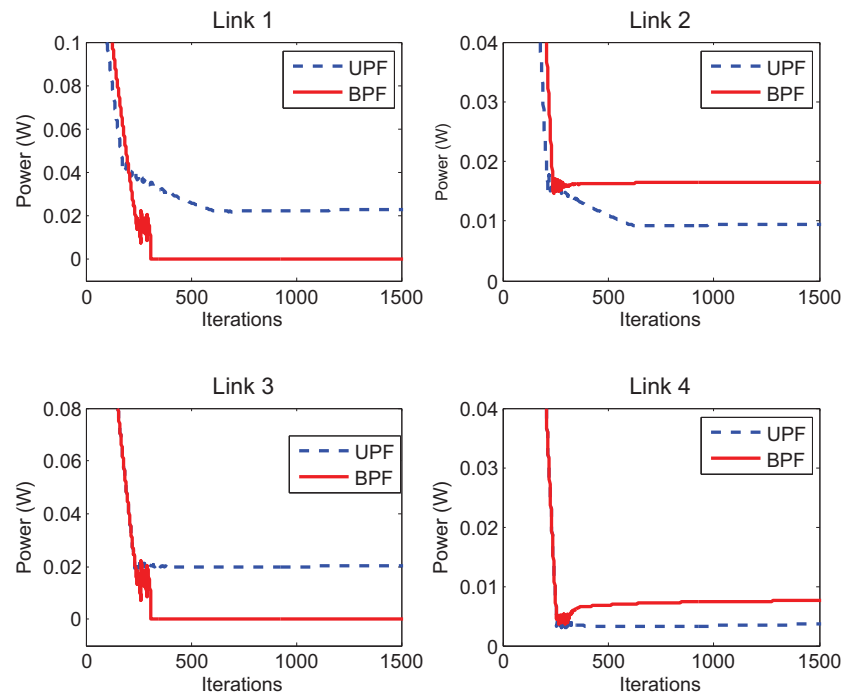

Fig. 5. Convergence of Transmission Power Allocation

\section{Concluding Remarks}

This paper discussed how utility proportional fairness can be used to resolve many of the shortcomings of traditional NUM approaches in wireless networks. More specifically, we proposed a utility proportional-fair optimization formulation for high-SINR wireless networks and developed a joint distributed rate and power allocation algorithm to solve this problem. In addition, it was shown that the use of utility proportional fairness allows the calculation of closed form solutions for the optimal rate allocation for a wide range of popular applications, prevents oscillations in the network and assures that all applications will be treated equally in terms of the rate allocation. Our approach was also simulated and compared against the traditional bandwidth proportional fair approach.
The focus of our future research will be twofold. First, we intend to examine more types of applications and calculate closed form solutions for the optimal rate allocation and, second, we will examine alternative approximations of the non-concave Shannon's capacity formula that will be efficient for both high and low SINR environments.

\section{REFERENCES}

[1] F. P. Kelly, A. Maulloo, and D. Tan, "Rate control in communication networks: Shadow prices, proportional fairness and stability," Journal of the Operational Research Society, pp. 237-252, 1998.

[2] S. Low and D. Lapsley, "Optimization flow control. i. basic algorithm and convergence," IEEE/ACM Transactions on Networking, vol. 7, no. 6, pp. 861 -874, December 1999.

[3] W. Stallings, Data and Computer Communications, 9th ed. Pearson Custom Publishing, 2010.

[4] D. Palomar and M. Chiang, "A tutorial on decomposition methods for network utility maximization," IEEE Journal on Selected Areas in Communications, vol. 24, no. 8, pp. 1439 -1451, August 2006.

[5] D. P. Palomar and M. Chiang, "Alternative distributed algorithms for network utility maximization: Framework and applications," $E E E$ Transactions on Automatic Control, vol. 52, no. 12, pp. $2254-2269$, December 2007.

[6] C. Liu, L. Shi, and B. Liu, "Utility-based bandwidth allocation for tripleplay services," in Universal Multiservice Networks, 2007. ECUMN '07. Fourth European Conference on, feb. 2007, pp. 327 -336.

[7] Cisco, "Cisco visual networking index: Forecast and methodology, 20102015," Cisco Systems Inc., Tech. Rep., June 2011. [Online]. Available: http://www.cisco.com/en/US/solutions/collateral/ ns341/ns525/ns537/ns705/ns827/white_paper_c11-520862.pdf

[8] J.-W. Lee, R. R. Mazumdar, and N. B. Shroff, "Non-convex optimization and rate control for multi-class services in the internet," IEEE Journal on Selected Areas in Communications, vol. 13, no. 4, pp. 827-840, August 2005.

[9] P. Hande, S. Zhang, and M. Chiang, "Distributed rate allocation for inelastic flows," IEEE/ACM Transactions on Networking, vol. 15, no. 6 , pp. $1240-1253$, December 2007.

[10] G. Tychogiogos, A. Gkelias, and K. K. Leung, "Towards a fair nonconvex resource allocation in wireless networks," in IEEE PIMRC 2011 Toronto, Canada, September 2011.

[11] W.-H. Wang, M. Palaniswami, and S. H. Low, "Application-oriented flow control: Fundamentals, algorithms and fairness," IEEE/ACM Transactions on Networking, vol. 14, no. 6, pp. 1282 -1291, December 2006.

[12] M. Chiang, "To layer or not to layer: balancing transport and physical layers in wireless multihop networks," in IEEE INFOCOM 2004, vol. 4, march 2004, pp. 2525 - 2536 vol.4.

[13] S. Boyd and L. Vandenberghe, Convex Optimization. Cambridge University Press, 2004

[14] D. P. Bertsekas, Nonlinear Programming. Athena Scientific, 1999.

[15] G. Tychogiogos, A. Gkelias, and K. K. Leung, "A new distributed optimization framework for hybrid ad-hoc networks," in IEEE GlobeCom 2011 - Workshop on Heterogeneous, Multi-Hop, Wireless and Mobile Networks, Houston, USA, December 2011.

[16] J. S. P. V. Hutson and M. J. Cloud, Applications of Functional Analysis and Operator Theory. Academic Press, 1980. 